\section{Quantum pharmacology}

Quantum Pharmacology. By W. G. Richards. Pp. xiii +213 . (Butterworth: Sevenoaks, UK, 1977.) £12.

THE relationship between the chemical structure of small molecules and their biological effects has been studied intensively for over a hundred years. Apart from its intrinsic interest, it is obviously of enormous practical importance in the design of drugs. In recent years, studies of this relationship have been growing steadily in sophistication. The biological effects can now be described in greater detail, often in terms of binding to isolatable receptors and enzymes rather than gross physiological effects. The structural properties of the small molecules, too, are now discussed with greater precision, considering their three-dimensional conformation and electron distribution rather than simply two-dimensional representations of their covalent structure. In the latter area, the past ten years have seen increasing application of molecular orbital methods to molecules of pharmacological interest, and Dr Richards, one of the leading practitioners of this interdisciplinary art, has now written an introduction to the field.

The stated aim of the book is twofold, to introduce medicinal chemists to molecular orbital calculations and to introduce theoreticians to the pharmacological problems to which their methods can be applied. Thus, the book begins with an outline (in 87 pages) of some basic pharmacology, lucidly presented though necessarily very condensed. The emphasis is primarily on the cholinergic and adrenergic systems, histamine and centrally acting drugs, with chemotherapy, prostaglandins and peptide hormones, for example, receiving only brief mention.

The second part (49 pages) introduces molecular orbital calculations. The fundamental properties of wave functions and molecular orbitals are described very clearly, as is their use to calculate molecular properties. There is a valuable chapter in which the various approximate wave functions are described and compared.

In many ways the core of the book is the third section ( 38 pages), as it is here that the applications of theory to experiment are discussed. The first topic is that of the conformation of the small molecule, illustrated by calculations on a number of neurotransmitters and, in particular, on a series of histamine derivatives. The latter illustrate the "essential conformation approach" developed by the author, in which the biological activity of a series of compounds is correlated with the region of conformational space which they can occupy, and a region of this space which is in some sense essential for activity is thus defined. Since most conformational calculations consider the small molecule in isolation (in vacuo), the next chapter is devoted to a discussion of approaches to the problem of including the effects of solvent (specifically water) in the calculations. The remaining chapters focus on electron distribution--both on empirical correlations with activity, and on the use of the electrostatic potential field of the small molecule as a method of "mapping" the complementary surface of the receptor site (introduced by Weinstein and his colleagues). It is a pity that this section of the book is so brief. It would have been valuable to have a more extensive discussion of some of the many interesting points raised, perhaps at the expense of a shorter pharmacological introduction, for which many introductory texts are available. The book concludes with a very valuable annotated bibliography of all molecular orbital studies of molecules of pharmacological interest reported up to the end of 1976.

\section{Remote sensing techniques}

Aerial Photography and Remote Sensing for Soil Survey. By L. P. White. Pp. viii +104 (Clarendon/ Oxford University: Oxford, 1977.) £6.

THE foreword to this book indicates that it is the first of a series of new handbooks following in the footsteps of Robin Clarke's classic The Study of the Soil in the Field. This is a disappointing book, mainly because it does not fulfil the expectations aroused by the title and preface. In short, the balance of the book seems to be out of keeping with the topic. Only one chapter (some eleven pages) is directly concerned with soil survey and aerial photography. The remainder of the main text of 93 pages consists of a concise discussion of remote sensing methods, sensors and platforms, which other texts have dealt with in a more comprehensive manner.

Readers concerned with the use of remote sensing techniques in soil survey will turn their attention to chapter 4. Those familiar with image interpretation will doubtless recognise the wide range of issues touched on here. The brevity of the treatment, however, results in a failure to discuss in any depth the many aspects of the relationships between soil mapping units and image characteristics. For example, the treatment of terrain analysis is rather perfunctory, and the topics of pedo-
Can calculations be useful? The author concludes with a cautious "yes", with which I am sure most readers will agree. The ways in which they are best used remains, to some extent, to be established. There is no question that molecular orbital calculations have revealed interesting correlations between molecular properties and biological activity-as, for example, in the "essential conformation approach" to histamine analogues-and these will continue to be a valuable aid to the rational design of new compounds. What we do not know is how such correlations can be interpreted in physical terms to help build up a picture of the receptor site. There is no doubt that further work in this field will be stimulated and guided by $\mathrm{Dr}$ Richards' lucid and critical description of the state of the art.

Gordon C. K. Roberts

Gordon C. K. Roberts is a member of the scientific staff of the Medical Research Council in the Division of Molecular Pharmacology, National Institute for Medical Research, London, UK.

logical analysis and photo-interpretation keys merit further discussion. A much fuller treatment of the value of images/photographs for the detection of temporal variations in soil conditions (for example, structure and profile drainage) would have been useful. Similarly, more attention could have been given to definitions of soil mapping units, their relationships to different scales of imagery, and the testing of the accuracy of interpretation.

The bulk of the book comprises descriptions of photography and photographic products, line-scanners, side looking radar, imagery from space platforms, image enhancement, and automatic analysis. There is, however, little significant comment on the sensors, platforms and techniques in relation to soil survey. Indeed, the illustrative plates have captions dealing with vegetation, relief and land use, rather than soil conditions or soil boundaries. Some plates are in colour.

A wider selection of references would have been appropriate in a volume of this kind. For example, the work of Beckett, Evans, Mitchell and Western would suggest some of the pathways towards greater use of remote sensing.

The line diagrams are good but there are some typographical errors such as incorrect naming of the general editors on the dust jacket.

L. F. Curtis

L. F. Curtis is Reader in Geography at the University of Bristol, $U K$. 